\title{
RELAÇÃO DA VELOCIDADE DE ESCOAMENTO DA SOLUÇÃO E DO COMPRIMENTO DA COLUNA DE SOLO COM OS PARÂMETROS DE TRANSPORTE DE POTÁSSIO EM UM LATOSSOLO E UM NEOSSOLO ${ }^{(1)}$
}

\author{
Danilo Pereira Ribeiro ${ }^{(2)}$, Mauro Aparecido Martinez $^{(3)}$, Antonio \\ Teixeira de Matos $^{(4)}$, Hugo Alberto Ruiz ${ }^{(5)}$, Marina Souza Nicácio \\ Parreiras $^{(6)}$ \& Paulo Roberto Cecon ${ }^{(7)}$
}

\begin{abstract}
RESUMO
Devido à importância dos estudos de deslocamento de solutos em colunas de solo, para avaliar os riscos de poluição do solo e das águas subterrâneas, e à falta de padronização no comprimento da coluna de solo utilizada (L) e na velocidade de escoamento da solução aplicada $(v)$, realizou-se este trabalho com o objetivo de avaliar a relação de $L$ e de $v$ com o coeficiente de dispersão-difusão (D), a dispersividade $(\lambda)$ e o fator de retardamento $(R)$ do $K^{+}$em um Latossolo Vermelho distrófico (LVd) e num Neossolo Quartzarênico órtico (RQo). Foram testados cinco comprimentos de colunas $(0,10,0,20,0,30,0,40$ e $0,50 \mathrm{~m})$ e quatro velocidades, sendo $0,62,0,69,0,75$ e $0,81 \mathrm{~m} \mathrm{~h}^{-1}$ para o LVd e 0,37, 0,40, 0,44 e $0,48 \mathrm{~m} \mathrm{~h}^{-1}$ para o RQo. Aplicouse uma solução deslocadora de $130 \mathrm{mg} \mathrm{L}^{-1} \mathrm{de} \mathrm{K}^{+}$nas colunas montadas com o solo desestruturado e saturadas com uma solução de $\mathrm{CaCl}_{2} 5 \mathrm{mmol} \mathrm{L}^{-1}$. Os parâmetros de transporte $\mathrm{R}$ e $\mathrm{D}$ foram obtidos utilizando-se o programa computacional Disp; $a \lambda$ foi obtida pela equação $D=D_{0}+\lambda_{v}$, sendo $D_{o}$ igual a $7,1310^{-6} \mathrm{~m}^{2} \mathrm{~h}^{-1}$ para o KCl. Houve relação do comprimento da coluna e da velocidade de escoamento da solução com os parâmetros de transporte do $\mathrm{K}^{+}$tanto no LVd quanto no RQo. No LVd, o fator de
\end{abstract}

\footnotetext{
(1) Parte da Dissertação de Mestrado do primeiro autor, apresentada ao Programa de Pós-Graduação em Engenharia Agrícola da Universidade Federal de Viçosa - UFV. Recebido para publicação em 2 de fevereiro de 2011 e aprovado em 15 de outubro de 2011.

${ }^{(2)}$ Mestrando do Programa de Pós-graduação em Eng. Agrícola, Universidade Federal de Viçosa - UFV. Av. P. H. Rolfs s/n, CEP 36570-000 Viçosa (MG). Bolsista do CNPq. E-mail: ribeirodp@bol.com.br

(3) Professor Titular do Departamento de Eng. Agrícola, DEA-UFV. Bolsista do CNPq. E-mail: mmauro@ufv.br

(4) Professor Associado do DEA-UFV. Bolsista do CNPq. E-mail: atmatos@ufv.br

(5) Professor Visitante Nacional Sênior/CAPES no Programa de Pós-Graduação em Produção Vegetal do Centro de Ciências Agrárias da Universidade Federal do Espírito Santo - CCA-UFES. Bolsista do CNPq. E-mail: hruiz@ufv.br

(6) Graduanda em Eng. Agrícola e Ambiental, UFV. E-mail: marinanicacio1@hotmail.com

(7) Professor Associado do Departamento de Estatística, DET-UFV. Bolsista do CNPq. E-mail: cecon@ufv.br
} 
retardamento aumentou com o aumento na velocidade de escoamento da solução. Em ambos os solos, o fator de retardamento diminuiu com o aumento no comprimento da coluna; o coeficiente de dispersão-difusão aumentou com o aumento na velocidade de escoamento da solução e com o aumento no comprimento da coluna de solo; e a dispersividade também aumentou com o aumento no comprimento da coluna.

Termos de indexação: deslocamento miscível, fator de retardamento, dispersividade, curva de eluição.

\title{
SUMMARY: RELATION OF THE PORE-WATER VELOCITY AND SOIL COLUMN LENGTH WITH POTASSIUM TRANSPORT PARAMETERS IN AN OXISOL AND A NEOSOL
}

\begin{abstract}
Due to the importance of studying solute displacement in soil columns to evaluate soil and groundwater pollution risks, and the lack of standardization in the soil column length $(L)$ and also the pore-water velocity ( $v)$ used, this study evaluated the relation of $L$ and $v$ with the dispersive-diffusive coefficient $(D)$, the dispersivity $(\lambda)$ and retardation factor $(R)$ of the potassium ion $\left(K^{+}\right)$in an Oxisol and in a Dystric Quartzarenic Neosol. Five column lengths were tested $(0.10,0.20,0.30,0.40$, and $0.50 \mathrm{~m})$ and four pore-water velocities $\left(0.62,0.69,0.75\right.$, and $0.81 \mathrm{~m} \mathrm{~h}^{-1}$ for the Oxisol and 0.37, 0.40, 0.44, and $0.48 \mathrm{~m} \mathrm{~h}^{-1}$ for the Neosol). A displacement solution of $130 \mathrm{mg} \mathrm{L}^{-1}$ of $\mathrm{K}^{+}$was applied to the disturbed soil columns saturated with a $5 \mathrm{mmol} \mathrm{L}^{-1} \mathrm{CaCl}_{2}$ solution. The transport parameters $R$ and $D$ were obtained using the computer program Disp; $\lambda$ was obtained by the equation $D=D_{o}+\lambda \mathrm{v}$, in which $D_{o}$ is equal to $7.1310^{-6} \mathrm{~m}^{2} \mathrm{~h}^{-1}$ for $\mathrm{KCl}$. The column length and pore-water velocity were related with parameters of the $K^{+}$transport in both soils. In the Oxisol, the retardation factor increased with increasing pore-water velocity. In both soils, the retardation factor decreased with increasing column length, the dispersivediffusive coefficient increased with increasing pore-water velocity and increasing soil column length and dispersivity also increased with increasing column length.
\end{abstract}

Index terms: Miscible Displacement, retardation factor, dispersivity, breakthrough curves.

\section{INTRODUÇÃO}

A preocupação com a poluição do solo e das águas subterrâneas tem resultado em inúmeros estudos de transporte de solutos, executados utilizando-se ensaios de deslocamento em colunas de solo (Bejat et al., 2000). Esses estudos são importantes ferramentas para se compreender o transporte de solutos adsorvidos e não adsorvidos pelo solo (Shukla et al., 2003). Muitos desses trabalhos analisaram o movimento de nutrientes ou metais pesados no solo utilizando soluções uni ou multiespécie (Matos et al., 1999; Araújo et al., 2003; Alcântara \& Camargo, 2010); outros foram desenvolvidos empregando diretamente as águas residuárias comumente aplicadas no solo (Dontsova et al., 2006; Ferreira et al., 2006; Anami et al., 2008). Vários estudos de deslocamento de substâncias químicas no solo, como agrotóxicos (Barizon et al., 2006; Montoya et al., 2006; Raymundo et al., 2009) e reguladores de crescimento vegetal (Milfont et al., 2008), são realizados utilizando colunas de lixiviação. O movimento de microrganismos no solo também tem sido estudado em ensaios com colunas (Chu et al., 2003; Guber et al., 2005; Rockhold et al., 2005).
Devido às dificuldades inerentes à coleta e ao preparo de colunas de solo estruturado (Corwin, 2000), bem como à grande variação dos resultados obtidos com esse material, o que implica grande quantidade de colunas para se obter representatividade (Akhtar et al., 2003), a maioria dos trabalhos é feita com solo passado em peneiras de 2 a $4 \mathrm{~mm}$.

Os resultados dos ensaios com colunas de lixiviação, aliados a modelos físico-matemáticos, proporcionam valiosos conhecimentos a respeito do meio poroso, como o comportamento dos solutos e processos associados, como difusão, dispersão, exclusão aniônica, sorção e mudanças durante o transporte (Shukla et al., 2003). De acordo com Costa \& Prunty (2006), a maioria das análises de transporte de solutos em meio poroso têm sido baseadas na equação de transporte convectivo-dispersivo desenvolvida por Lapidus \& Amundson (1952).

Apesar de os trabalhos de deslocamento de solutos utilizarem a mesma equação de transporte, algumas condições experimentais - como o comprimento da coluna, a velocidade de deslocamento da solução aplicada e a maneira como a coluna foi preenchida 
com solo - não têm uma devida padronização, o que dificulta a comparação dos resultados entre diferentes trabalhos (Bromly et al., 2007; Lewis \& Sjöstrom, 2010). Segundo Lewis \& Sjöstrom (2010), as dimensões das colunas de lixiviação relatadas na literatura variam de $1,4 \mathrm{~cm}$ de comprimento e $1 \mathrm{~cm}$ de diâmetro, nas menores colunas, a $2 \times 2 \times 5 \mathrm{~m}$, nas maiores.

Acredita-se que os parâmetros de transporte de solutos obtidos em experimentos de laboratório sejam dependentes das condições experimentais (Bromly et al., 2007). Para a condição de campo ou em escalas de aquífero, está bem estabelecido que a dispersividade $(\lambda)$ aumenta com o aumento do percurso (Butters \& Jury, 1989; Gelhar et al., 1992; Basha \& El-Habel, 1993). No entanto, resultados de estudos com colunas em laboratório em que foi avaliado o efeito do comprimento da coluna sobre a dispersividade são inconsistentes. Alguns estudos reportam o aumento da dispersividade com o aumento do comprimento da coluna (Wierenga \& van Genuchten, 1989; Huang et al., 1995; Li \& Godrati, 1995); outros relatam que a dispersividade permaneceu inalterada, comparandose os resultados para as colunas com diferentes comprimentos (Khan \& Jury, 1990; Shukla et al., 2003). Bromly et al. (2007) analisaram vários trabalhos com colunas de lixiviação e observaram que a dispersividade foi maior para as colunas de até $10,7 \mathrm{~cm}$ de comprimento.

A variação na velocidade de escoamento aplicada nas colunas de lixiviação pode alterar os outros parâmetros da equação de transporte (Álvarez-Benedí, 1999). A influência da velocidade sobre o coeficiente de dispersão-difusão (D) tem sido bem relatada na literatura (Lewis \& Sjöstrom, 2010). A influência da velocidade de escoamento sobre o fator de retardamento de soluto (R) também tem sido relatada na literatura; no entanto, os resultados são inconsistentes. Shukla et al. (2003) e Wierenga \& van Genuchten (1989) observaram aumento no $\mathrm{R}$ do ânion cloreto com o aumento na velocidade, ao passo que Nkedi-Kizza et al. (1983) e Schulin et al. (1987) relataram diminuição no $R$ com o aumento na velocidade.

Assim, observa-se que os parâmetros de transporte de solutos são influenciados pelas condições experimentais, como o comprimento da coluna e a velocidade de escoamento da solução, porém essas relações ainda não são claras. Em quase todos os trabalhos, os parâmetros de transporte de solutos, como o coeficiente de dispersão-difusão e a dispersividade, obtidos empregando solutos não reativos, são utilizados para modelar condições de transporte reativo (Brusseau, 1993), o que torna sua aplicação questionável.

Diante do exposto, este trabalho foi realizado com o objetivo de avaliar a relação da velocidade de escoamento da solução e do comprimento da coluna de solo com o coeficiente de dispersão-difusão, a dispersividade e o fator de retardamento do $\mathrm{K}^{+} \mathrm{em}$ um Latossolo Vermelho distrófico (LVd) e em um Neossolo Quartzarênico órtico (RQo).

\section{MATERIAL E MÉTODOS}

Os solos utilizados foram um Neossolo Quartzarênico ortico (RQo), proveniente da cidade de Três Marias, e um Latossolo Vermelho distrófico (LVd), proveniente da cidade de Sete Lagoas, em Minas Gerais, cujas amostras foram coletadas na camada de 0 a $20 \mathrm{~cm}$, secas ao ar e passadas em peneiras de $2 \mathrm{~mm}$.

As análises de caracterização física dos solos foram feitas utilizando-se o método proposto por Ruiz (2005), para a análise granulométrica, e os métodos relacionados pela Embrapa (1997), para as determinações de densidade do solo e de partícula, porosidade total e condutividade hidráulica. As análises químicas foram realizadas de acordo com os métodos apresentados em Defelipo \& Ribeiro (1997). Os resultados dessas análises são apresentados no quadro 1.

Foram testados, para cada solo, cinco dos principais comprimentos observados em trabalhos com colunas de lixiviação $(0,10,0,20,0,30,0,40$ e $0,50 \mathrm{~m})$ e quatro diferentes velocidades de escoamento. Os experimentos foram instalados num esquema em parcelas subdivididas, tendo as velocidades de escoamento ( $v$ ) como parcelas e os comprimentos das

Quadro 1. Caracterização física e química do Latossolo Vermelho distrófico (LVd) e do Neossolo Quartzarênico órtico (RQo)

\begin{tabular}{|c|c|c|}
\hline Característica & LVd & RQo \\
\hline Areia grossa $\left(\mathrm{g} \mathrm{kg}^{-1}\right)$ & 70 & 220 \\
\hline Areia fina $\left(\mathrm{g} \mathrm{kg}^{-1}\right)$ & 50 & 600 \\
\hline Silte $\left(\mathrm{g} \mathrm{kg}^{-1}\right)$ & 60 & 30 \\
\hline Argila $\left(\mathrm{g} \mathrm{kg}^{-1}\right)$ & 820 & 150 \\
\hline Densidade do solo $\left(\mathrm{kg} \mathrm{dm}^{-3}\right)$ & 0,84 & 1,37 \\
\hline Densidade de partículas $\left(\mathrm{kg} \mathrm{dm}^{-3}\right)$ & 2,62 & 2,63 \\
\hline Porosidade total $\left(\mathrm{m}^{3} \mathrm{~m}^{-3}\right)$ & 0,68 & 0,48 \\
\hline Cond. hidráulica saturada $\left(\mathrm{m} \mathrm{h}^{-1}\right)^{(1)}$ & 0,375 & 0,13 \\
\hline pH em água $(1: 2,5)$ & 4,37 & 4,25 \\
\hline $\mathrm{pH}$ em $\mathrm{KCl}(1: 2,5)$ & 4,14 & 4,01 \\
\hline Potássio trocável $\left(\mathrm{cmol}_{\mathrm{c}} \mathrm{dm}^{-3}\right)$ & 0,068 & 0,028 \\
\hline Sódio trocável $\left(\mathrm{cmol}_{\mathrm{c}} \mathrm{dm}^{-3}\right)$ & 0,0056 & 0,0056 \\
\hline Cálcio trocável $\left(\mathrm{cmol}_{\mathrm{c}} \mathrm{dm}^{-3}\right)$ & 0,95 & 0,08 \\
\hline Magnésio trocável $\left(\mathrm{cmol}_{\mathrm{c}} \mathrm{dm}^{-3}\right)$ & 0,06 & 0,02 \\
\hline Alumínio trocável $\left(\mathrm{cmol}_{\mathrm{c}} \mathrm{dm}^{-3}\right)$ & 1,43 & 0,00 \\
\hline $\mathrm{H}+\mathrm{Al}\left(\mathrm{cmol}_{\mathrm{c}} \mathrm{dm}^{-3}\right)$ & 11,2 & 3,4 \\
\hline Soma de bases trocáveis $\left(\mathrm{cmol}_{\mathrm{c}} \mathrm{dm}^{-3}\right)$ & 1,08 & 0,13 \\
\hline CTC efetiva $\left(\mathrm{cmol}_{\mathrm{c}} \mathrm{dm}^{-3}\right)$ & 2,51 & 0,13 \\
\hline CTC a pH 7,0 $\left(\mathrm{cmol}_{\mathrm{c}} \mathrm{dm}^{-3}\right)$ & 12,28 & 3,53 \\
\hline Índice de saturação por bases (\%) & 8,8 & 3,7 \\
\hline Índice de saturação por alumínio (\%) & 57 & 0,0 \\
\hline Fósforo disponível $\left(\mathrm{mg} \mathrm{dm}^{-3}\right)$ & 2,8 & 3,3 \\
\hline Carbono orgânico $\left(\mathrm{g} \mathrm{kg}^{-1}\right)$ & 62,1 & 7,6 \\
\hline
\end{tabular}

(1) Médias de todas as parcelas corrigidas para $20^{\circ} \mathrm{C}$. Mat. Org. $=$ C.Org. x 1,724 - Walkley-Black; P, Na e K: extrator Mehlich-1 $1 \mathrm{~mol} \mathrm{~L}^{-1} ; \mathrm{Ca}, \mathrm{Mg}$ e Al - extrator $\mathrm{KCl}, 1 \mathrm{~mol} \mathrm{~L}^{-1} ; \mathrm{H}+\mathrm{Al}$ : extrator acetato de cálcio $0,5 \mathrm{~mol} \mathrm{~L}^{-1} ; \mathrm{pH}$ 7; Dens. do solo pelo método da proveta e de partícula pelo método do balão. 
colunas (L) como subparcelas. O delineamento experimental foi em blocos casualizados (DBC), com três repetições.

As colunas de lixiviação foram confeccionadas com tubos de PVC, com diâmetro interno de $4,7 \mathrm{~cm}$ e comprimentos proporcionais aos tratamentos, mais uma folga para manter a carga hidráulica. Areia foi colada em toda a parte interna do tubo, a fim de evitar o escoamento preferencial pelas paredes. $\mathrm{Na}$ extremidade inferior das colunas foram fixadas telas de malha fina $(\leq 2 \mathrm{~mm})$ e, acima da tela, um disco de lã de vidro, para evitar a perda de partículas.

Os volumes internos correspondentes aos cinco comprimentos foram determinados, e a quantidade de solo colocada em cada coluna proporcionou a mesma densidade (Quadro 1) para todas as colunas de um mesmo tipo de solo. Essas quantidades foram colocadas nas colunas em camadas de $2 \mathrm{~cm}$, misturando sempre a camada anterior com a subseqüente, com o auxílio de um garfo. A acomodação do solo era feita periodicamente, com toques de dedos em torno do tubo. Depois de obtido o comprimento desejado, foi colocado um disco de lã de vidro para evitar distúrbios na superfície do solo ao interligar a coluna com a fonte de água (solução $\mathrm{CaCl}_{2}$ ) ou solução de $\mathrm{KCl}$.

O volume de poros para cada tipo de solo e comprimento de coluna foi determinado conforme apresentado por Ruiz et al. (2010).

As colunas com solo foram saturadas de forma ascendente, por um período mínimo de $24 \mathrm{~h}$, com uma solução de $\mathrm{CaCl}_{2} 5 \mathrm{mmol} \mathrm{L}{ }^{-1}$, utilizada com o intuito de desfavorecer a dispersão de argila. Terminada a saturação, as colunas foram conectadas aos frascos de Mariotte, aplicando-se a solução de $\mathrm{CaCl}_{2}$ até obtenção de escoamento permanente. O procedimento experimental foi realizado conforme descrito em Ruiz et al. (2010).

A condutividade hidráulica do solo saturado foi determinada nas próprias colunas de lixiviação, utilizando-se o método do permeâmetro de carga constante (Embrapa, 1997). As diferentes velocidades aplicadas foram obtidas a partir da condutividade hidráulica média de 10 colunas (duas para cada comprimento de coluna) para cada solo.

Após a determinação da condutividade hidráulica, as cargas de pressão nas superfícies do solo das colunas eram estabelecidas de modo que fosse obtida uma das quatro velocidades aplicadas, que foram de $0,37,0,40$, 0,44 e $0,48 \mathrm{~m} \mathrm{~h}^{-1}$ para o RQo e de $0,62,0,69,0,75$ e $0,81 \mathrm{~m} \mathrm{~h}^{-1}$ para o LVd. Os tratamentos foram definidos pretendendo-se incrementos de $10 \%$ na velocidade de escoamento da solução.

Após atingir o escoamento permanente na coluna de lixiviação, a solução de $\mathrm{CaCl}_{2}$ foi substituída pela solução de $\mathrm{KCl}$ com concentração $\mathrm{C}_{\mathrm{o}}=130 \mathrm{mg} \mathrm{L}^{-1} \mathrm{de}$ $\mathrm{K}^{+}$. Esta solução foi aplicada até que o volume percolado correspondesse a sete volumes de poros para o LVd e cinco volumes de poros para o RQo (Oliveira et al., 2004). Na extremidade inferior da coluna, foram coletados volumes de efluente, divididos em 18 intervalos de mesmo volume para cada comprimento de coluna, que corresponderam a aproximadamente 0,28 e 0,39 volumes de poros para o Neossolo e o Latossolo, respectivamente. Posteriormente, retiravase uma alíquota, que era acondicionada em recipiente de vidro, fechado hermeticamente, e guardada em freezer para análise posterior.

As amostras da solução efluente de $\mathrm{K}$ foram analisadas por fotometria de emissão de chama, obtendo-se assim a concentração de K efluente (C) e a concentração relativa $\left(\mathrm{C} / \mathrm{C}_{0}\right)$. As concentrações relativas e os respectivos números de volumes de poros foram utilizados para elaborar as curvas experimentais de efluente de cada tratamento.

Os parâmetros de transporte $\mathrm{R}$ e $\mathrm{D}$ foram obtidos utilizando-se o programa computacional Disp (Borges Júnior \& Ferreira, 2006), usando-se o modelo A-1, o qual ajusta os dados experimentais a soluções matemáticas da equação diferencial de transporte de solutos no solo (Wierenga \& van Genuchten, 1989) com base na menor soma dos mínimos quadrados dos resíduos. O programa considera a isoterma de sorção linear para se determinar o fator de retardamento.

Os dados de fluxo de Darcy, porosidade total, comprimento da coluna de solo, número de volumes de poros, densidade do solo e $\mathrm{C} / \mathrm{C}_{0}$ foram as variáveis de entrada no DISP. A dispersividade foi calculada usando a equação $\lambda=\left(\mathrm{D}-\mathrm{D}_{0}\right) / v$, em que $\mathrm{D}_{0}$ é a difusão do $\mathrm{K}$ em água pura, considerada como sendo de $7,1310^{-6} \mathrm{~m}^{2} \mathrm{~h}^{-1}$ para o $\mathrm{KCl}$ (Lobo et al., 1998).

Os valores de $\mathrm{D}, \mathrm{R}$ e $\lambda$ obtidos operando-se os permeâmetros sob as quatro magnitudes de fluxo e os cinco comprimentos das colunas de cada solo foram submetidos à análise de variância e de regressão, utilizando-se para isso o software SAEG 9.1. Os modelos foram escolhidos com base na significância dos coeficientes de regressão, utilizando-se o teste $t$, no coeficiente de determinação e na análise de resíduo.

\section{RESULTADOS E DISCUSSÃO}

As velocidades de escoamento da solução observadas para o solo argiloso (LVd) foram elevadas; contudo, altas velocidades têm sido relatadas em trabalhos com colunas de lixiviação preenchidas com Latossolos. Matos et al. (1999) obtiveram velocidade média da solução em um Latossolo Vermelho-Amarelo de $1,12 \mathrm{~m} \mathrm{~h}^{-1}$. Oliveira et al. (2004) apresentaram velocidades médias da solução em três Latossolos Vermelhos distróficos e um Latossolo Vermelho-Amarelo de 2,95, 1,43, 2,40 e $1,71 \mathrm{~m} \mathrm{~h}^{-1}$, respectivamente. Segundo esses autores, a estrutura granular dos Latossolos propicia elevada porosidade, principalmente de macroporos, o que favorece o desenvolvimento de altas velocidades de escoamento nestes solos. 
A macroporosidade dos solos avaliados, estimada em função da densidade do solo e do teor de areia pela equação proposta por Stolf et al. (2011), foi de $0,328 \mathrm{~m}^{3} \mathrm{~m}^{-3}$ para o LVd e de $0,23 \mathrm{~m}^{3} \mathrm{~m}^{-3}$ para o RQo. A equação, de alto coeficiente de determinação, foi obtida para solos muito semelhantes aos utilizados neste trabalho. Oliveira et al. (2004) avaliaram o mesmo Latossolo (LVd de Sete Lagoas, MG) e obtiveram idêntica densidade do solo e teor de areia; a macroporosidade determinada foi de $0,317 \mathrm{~m}^{3} \mathrm{~m}^{-3}$, muito próxima da estimada no presente trabalho. Como a condutividade hidráulica é influenciada principalmente pela macroporosidade, esses resultados e a porosidade total dos solos (Quadro 1) justificam as maiores velocidades de escoamento da solução obtidas no LVd em relação ao RQo.

A influência do comprimento da coluna no fator de retardamento foi constatada pelo teste $t$, demonstrando que houve relação linear simples negativa do fator de retardamento com o comprimento da coluna, como pode ser observado na figura 1. A diminuição do fator de retardamento pode ser explicada pela diminuição no coeficiente de distribuição, com o aumento do comprimento da coluna de solo, já que os outros termos que descrevem o fator de retardamento não variaram com o comprimento da coluna. A influência do comprimento da coluna sobre o fator de retardamento não foi observada em outros trabalhos que avaliaram o efeito do comprimento da coluna sobre os parâmetros de transporte de solutos (Wierenga \& van Genuchten, 1989; Shukla et al., 2003); contudo, esses trabalhos foram feitos com solutos não reativos e tiveram menor número de avaliações.

O aumento da coluna de solo contribui para diminuir a uniformidade no preenchimento da coluna, e isso, aliado ao efeito da gravidade, pode provocar regiões com maior compactação do solo próximo à base da coluna, o que ocasiona zonas com menor mobilidade

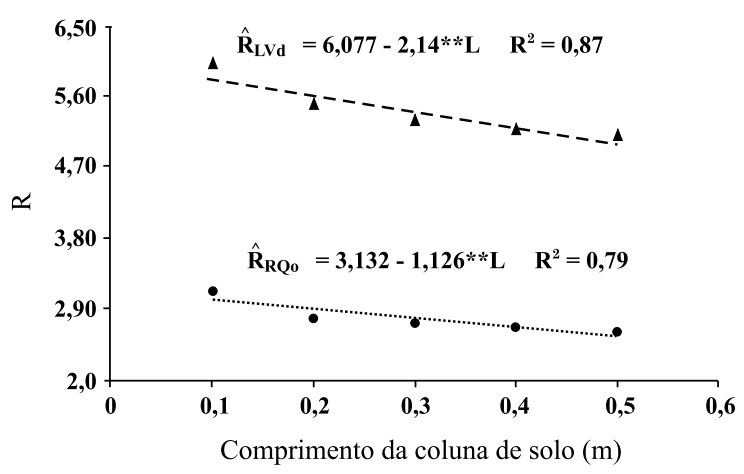

Figura 1. Fator de retardamento (R) de potássio estimado (linhas) e médias dos valores experimentais observados no Latossolo Vermelho distrófico (LVd) (ム) e no Neossolo Quartzarênico órtico (RQo) (O), em função do comprimento da coluna de solo $(\mathrm{L}) .{ }^{* *}$ : significativo a $1 \%$, pelo teste $t$. da solução ou até mesmo com conteúdo de água imóvel, onde a adsorção e dessorção do soluto ocorrem apenas por difusão. Por outro lado, como as colunas apresentam a mesma densidade do solo, há regiões nas colunas maiores, com poros maiores, que favorecem o aumento da velocidade de escoamento nesses poros e o menor tempo de interação entre o solo e o soluto. Esse menor tempo de interação solosoluto e a diminuição do movimento do soluto pelos mecanismos de dispersão e fluxo em massa nas zonas compactadas favorecem a diminuição do fator de retardamento.

Os valores do fator de retardamento estimados para o LVd, pela equação da figura 1, diminuíram de 5,86 para 5,01 para as colunas de 0,10 a $0,50 \mathrm{~m}$, respectivamente. Os valores estimados de R em função do comprimento da coluna para o RQo diminuíram de 3,02 para a coluna de $0,10 \mathrm{~m}$ para 2,57 na coluna de $0,50 \mathrm{~m}$. Esses valores estão entre os observados por Silva (2009) para um Argissolo Vermelho-Amarelo eutrófico (PVAe) típico caulinítico fase terraço e um Latossolo Vermelho-Amarelo distrófico (LVAd), que apresentaram fatores de retardamento médios para o $\mathrm{K}$ de 6,43 e 1,72, respectivamente. As colunas preenchidas com os solos desestruturados tinham $0,20 \mathrm{~m}$ de comprimento e velocidade de $0,31 \mathrm{e}$ $0,115 \mathrm{~m} \mathrm{~h}^{-1}$ para o PVAe e LVAd, respectivamente.

Analisando o movimento de K em água residuária de manipueira em três tipos de solo, Melo et al. (2006) obtiveram fatores de retardamento de 1,52 para um Latossolo Amarelo distrófico (LAd), de 2,39 para um Latossolo Vermelho-Amarelo distrófico (LVAd) e de 1,18 para um Neossolo Quartzarênico órtico espódico (RQo). As colunas utilizadas foram de $0,30 \mathrm{~m}$ de comprimento, e as velocidades médias, de 18,5, 3 e $24 \mathrm{~cm} \mathrm{~h}^{-1}$ para o LAd, LVAd e RQo, respectivamente.

Ferreira et al. (2006) avaliaram o movimento de K na água residuária de café conilon em colunas de solo, utilizando colunas de $0,20 \mathrm{~m}$, e relataram $\mathrm{R}$ de 1,27 para um Argissolo Vermelho-Amarelo distrófico (PVAd) com velocidade de $17 \mathrm{~cm} \mathrm{~h}^{-1}$, de 2,58 para um Latossolo Vermelho-Amarelo eutrófico (LVAe) com velocidade de $13 \mathrm{~cm} \mathrm{~h}^{-1}$ e de 1,22 para um solo aluvial eutrófico com velocidade da solução de $21 \mathrm{~cm} \mathrm{~h}^{-1}$.

Oliveira et al. (2004) avaliaram o movimento de K no mesmo LVd estudado no presente trabalho, variando a concentração de $\mathrm{K}, \mathrm{P}$ e $\mathrm{NH}_{4}{ }^{+}$na solução deslocadora aplicada em colunas de 0,20 m. Para esse comprimento, o fator de retardamento estimado pela equação da figura 1 foi de 5,65. Esse valor está acima da média de 4,18 obtida pelos autores, porém está dentro do intervalo de 2,94 a 6,57 observado para o LVd. Esses autores avaliaram também o movimento de $\mathrm{K}$ num RQo e verificaram que os resultados de $\mathrm{R}$ variaram de 3,93 a 2,46 , com média de 3,16 , ao passo que o valor estimado de acordo com a equação da figura 1 foi de 2,91 .

$\mathrm{O}$ fator de retardamento do $\mathrm{K}$ foi maior no solo argiloso devido à maior capacidade de troca catiônica, 
favorecida pela maior quantidade de argila e também de matéria orgânica nesse solo (Quadro 1). Esse resultado é semelhante aos de Oliveira et al. (2004), Ferreira et al. (2006) e Melo et al. (2006), que também observaram maior fator de retardamento do $\mathrm{K}$ nos solos mais argilosos.

A velocidade de escoamento também apresentou influência significativa sobre o fator de retardamento do K no LVd, com ajuste de regressão linear simples positiva (Figura 2).

$\mathrm{O}$ aumento do fator de retardamento com a velocidade, apesar de pequeno, não era esperado. No entanto, essa relação já foi observada em outros trabalhos. Shukla et al. (2003) observaram aumento do $\mathrm{R}$ do $\mathrm{Cl}^{-}$de 0,8 para 1,2 com o aumento da velocidade para a condição de solo saturado, o que atribuíram à diminuição da região de exclusão aniônica com o aumento da velocidade. Wierenga \& van Genuchten (1989) também observaram aumento do $\mathrm{R}$ do $\mathrm{Cl}^{-}$, assim como do $\mathrm{R}$ do cátion trítio $\left({ }^{3} \mathrm{H}^{+}\right)$, com o aumento da $v$. Para o cátion ${ }^{3} \mathrm{H}^{+}$não foram apresentadas justificativas para esse resultado, mas para o $\mathrm{Cl}^{-}$foi justificada a diminuição da região de exclusão aniônica.

Conforme mencionado, o aumento da coluna de solo pode ter propiciado o aumento de regiões com conteúdo de água imóvel, as quais diminuem a quantidade de solo ou área superficial específica, disponível para os processos de sorção. O aumento da velocidade de escoamento da solução aumenta o efeito da dispersão e do fluxo em massa, fazendo com que a solução deslocadora penetre nessas regiões com água imóvel, aumentando a quantidade de solo disponível para os processos de sorção. Isso pode favorecer o incremento do fator de retardamento com o aumento da velocidade de escoamento da solução, como observado no LVd. Como esse aumento do fator de retardamento no LVd, que é o solo mais reativo, foi muito pequeno, o mesmo efeito no RQo pode não ter favorecido resultados significativos.

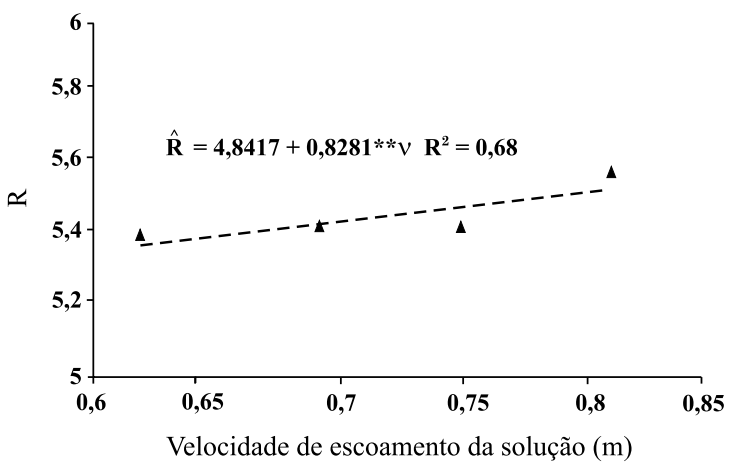

Figura 2. Fator de retardamento (R) de potássio estimado (- - ) e médias dos valores experimentais $(\Delta)$ observados num Latossolo Vermelho distrófico (LVd), em função da velocidade de escoamento da solução $(v)$. **: significativo a $1 \%$ pelo teste $t$.
A diminuição do fator de retardamento com o aumento da velocidade também foi observada em alguns trabalhos. Schulin et al. (1987) observaram esse efeito para os íons ${ }^{3} \mathrm{H}^{+}$e Br- . Nkedi-Kizza et al. (1983) também encontraram menores fatores de retardamento para $\mathrm{Cl}^{-} \mathrm{e}^{3} \mathrm{H}^{+}$com o aumento da velocidade, o que atribuíram ao menor tempo de residência do soluto na solução do solo à medida que a velocidade aumenta, provocando menor transferência de massa por difusão para dentro e fora da região com água imóvel. As velocidades observadas por esses autores variaram de 4,4 a $96,5 \mathrm{~mm} \mathrm{~h}^{-1}$, sendo bem menores que as observadas no presente trabalho, que têm, portanto, maior capacidade de provocar dispersão e fluxo em massa do soluto para regiões com menor mobilidade.

O coeficiente de dispersão-difusão de K apresentou relação linear múltipla em função da velocidade de escoamento e do comprimento da coluna de solo, tanto para o LVd (Figura 3) quanto para o RQo (Figura 4). A relação linear da velocidade de escoamento da solução com o D tem sido relatada em vários trabalhos com colunas de lixiviação (Álvarez-Benedí et al., 1999; Shukla et al., 2003; Bromly et al., 2007; Lewis \& Sjöstrom, 2010).

Wierenga \& van Genuchten (1989) também avaliaram a influência do comprimento da coluna nos parâmetros de transporte, utilizando para isso uma coluna com $6 \mathrm{~m}$ de comprimento e coletando amostras em várias profundidades. Esses autores não observaram relação clara do D com o comprimento da coluna, pois este aumentou até $4 \mathrm{~m}$, porém diminuiu aos $5 \mathrm{~m}$ de profundidade.

Biggar \& Nielsen (1976) e Jaynes et al. (1988), avaliando o movimento de solutos num experimento em campo sob condição de irrigação por inundação, coletando amostras em várias profundidades $(0,3 \mathrm{a}$ $1,83 \mathrm{~m}$ e 0,3 a 3,0 m, respectivamente), encontraram relação aproximadamente linear do $\mathrm{D}$ com a velocidade, mas não observaram relação do $\mathrm{D}$ com a profundidade amostrada.

No LVd, os valores estimados do coeficiente de dispersão-difusão variaram de $87 \mathrm{~cm}^{2} \mathrm{~h}^{-1}$ na coluna de $0,10 \mathrm{~m}$ e velocidade de $0,62 \mathrm{~m} \mathrm{~h}^{-1}$ a $277 \mathrm{~cm}^{2} \mathrm{~h}^{-1}$ na coluna de $0,50 \mathrm{~m}^{2}$ velocidade de $0,81 \mathrm{~m} \mathrm{~h}^{-1}$. Para o RQo, os valores de D estimados variaram de $48 \mathrm{~cm}^{2} \mathrm{~h}^{-1}$, para a velocidade de $0,37 \mathrm{~m} \mathrm{~h}^{-1} \mathrm{e}$ comprimento da coluna de $0,10 \mathrm{~m}$, a $183 \mathrm{~cm}^{2} \mathrm{~h}^{-1}$, para a velocidade de $0,48 \mathrm{~m} \mathrm{~h}^{-1}$ e comprimento da coluna de $0,50 \mathrm{~m}$. Os maiores valores do coeficiente de dispersão-difusão, observados no LVd, podem ser atribuídos aos maiores valores de velocidade de escoamento da solução desenvolvidos neste solo.

Os coeficientes de dispersão-difusão de Kobservados por Oliveira et al. (2004) para o LVd e um RQo foram bem maiores que os observados neste e em outros trabalhos, com média de $0,348 \mathrm{~m}^{2} \mathrm{~h}^{-1}$ no LVd e de $0,292 \mathrm{~m}^{2} \mathrm{~h}^{-1}$ num RQo; contudo, as velocidades de escoamento das soluções aplicadas também foram 


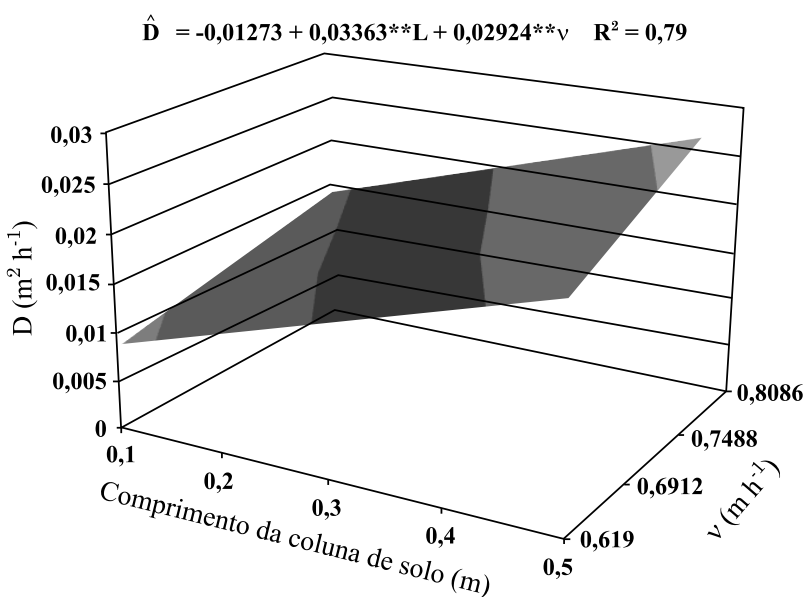

Figura 3. Estimativas do coeficiente de dispersãodifusão (D) de potássio num Latossolo Vermelho distrófico (LVd), em função de colunas de solo com 0,10 a $0,50 \mathrm{~m}$ de comprimento (L) e de velocidades de escoamento da solução $(v)$ de 0,62 a $0,81 \mathrm{~m} \mathrm{~h}^{-1}$.**: significativo a $1 \%$ pelo teste $t$.

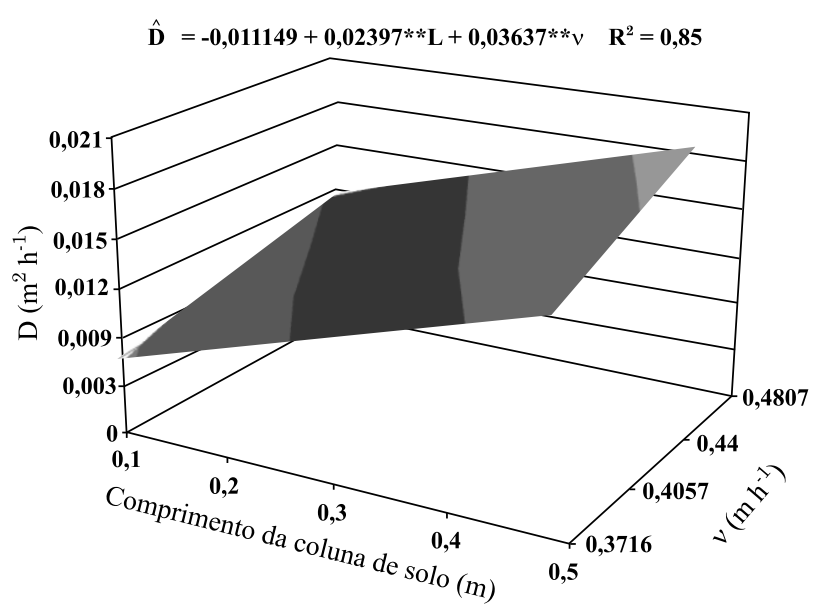

Figura 4. Estimativas do coeficiente de dispersãodifusão (D) de potássio num Neossolo Quartzarênico órtico (RQo), em função de colunas de solo com 0,10 a $0,50 \mathrm{~m}$ de comprimento (L) e de velocidades de escoamento da solução (v) de 0,37 a $0,48 \mathrm{~m} \mathrm{~h}^{-1}$. **: significativo a $1 \%$ pelo teste $t$.

maiores. Silva (2009) relatou coeficiente dispersivodifusivo de $29 \mathrm{~cm}^{2} \mathrm{~h}^{-1}$ para o PVAe e de $11,5 \mathrm{~cm}^{2} \mathrm{~h}^{-1}$ para o LVAd. Melo et al. (2006) relataram valores de $31 \mathrm{~cm}^{2} \mathrm{~h}^{-1}$ para o LAd, de $6 \mathrm{~cm}^{2} \mathrm{~h}^{-1}$ para o LVAd e de $125 \mathrm{~cm}^{2} \mathrm{~h}^{-1}$ para o RQo. Ferreira et al. (2006) apresentaram valores de $7 \mathrm{~cm}^{2} \mathrm{~h}^{-1}$ para o solo aluvial, de $13 \mathrm{~cm}^{2} \mathrm{~h}^{-1}$ para o PVAd e de $2,6 \mathrm{~cm}^{2} \mathrm{~h}^{-1}$ para o LVAe.

O coeficiente de dispersão-difusão depende da dispersividade, do coeficiente de difusão do soluto em água e da velocidade de escoamento da solução; para uma mesma velocidade aplicada, o coeficiente de difusão do soluto em água não se altera, variam-se apenas os comprimentos das colunas utilizadas. A influência do comprimento da coluna de lixiviação sobre o parâmetro D pode ser atribuída ao aumento na dispersividade, que é uma propriedade intrínseca do solo (Lewis \& Sjöstrom, 2010).

A dispersividade do K no LVd e no RQo apresentou ajuste de regressão linear simples em função do comprimento da coluna de solo, tal como podem ser observadas na figura 5 . Não houve ajuste de regressão da dispersividade em função da velocidade de escoamento da solução. Esse resultado era esperado, pois a dispersividade deve ser constante quando o coeficiente de dispersão-difusão for função da velocidade. De acordo com Bromly et al. (2007), a dispersividade é geralmente considerada uma propriedade intrínseca do solo, por ser dependente do meio poroso e independente da $v$.

A dispersividade estimada em função do comprimento da coluna de solo, para o LVd, de acordo com a figura 5 , aumentou de $16,4 \mathrm{~mm}$, na coluna de $0,10 \mathrm{~m}$, para $34,5 \mathrm{~mm}$, na coluna de $0,50 \mathrm{~m}$. Para o RQo, a dispersividade estimada aumentou de $15,7 \mathrm{~mm}$, na coluna de $0,10 \mathrm{~m}$, para $38 \mathrm{~mm}$, na coluna de $0,50 \mathrm{~m}$. Alguns trabalhos com colunas de solo ou em campo também relataram esse efeito.

Segundo Wierenga \& van Genuchten (1989), a dispersividade do $\mathrm{Cl}^{-}$variou de aproximadamente $1 \mathrm{~cm}$ em colunas com 0,30 m de comprimento a $5 \mathrm{~cm}$ em uma coluna de $6 \mathrm{~m}$ de comprimento. Huang et al. (1995) também observaram variações na dispersividade do $\mathrm{NaCl}$ de 1 a $5 \mathrm{~cm}$ para uma coluna de solo arenoso desestruturado, saturado, de 12,5 m de comprimento e montada na posição horizontal.

Jaynes et al. (1988) encontraram relação linear positiva da dispersividade com a profundidade do solo, a qual variou de 0,3 a $3,0 \mathrm{~m}$, apesar de o $\mathrm{D}$ não ter sido influenciado significativamente pela profundidade. Esses autores relataram que a velocidade diminuía com a profundidade, enquanto o D aumentava.

Estudando a influência da velocidade e da profundidade do solo nos parâmetros de transporte de solutos, utilizando o Br" como traçador, Costa \& Prunty (2006), em experimento em campo sob solo saturado, observaram, em condição de velocidade maior que $21,4 \mathrm{~mm} \mathrm{~h}^{-1}$, que a dispersividade aumentou de $4,9 \mathrm{~mm}$ aos $0,30 \mathrm{~m}$ de profundidade para $23,8 \mathrm{~mm}$ aos 2,50 $\mathrm{m}$ de profundidade.

$\mathrm{O}$ aumento da $\lambda$ com a profundidade do solo ou com o comprimento da coluna pode ser causado pela maior heterogeneidade macroscópica do meio poroso, em vez de ser pela heterogeneidade em escala dos poros (Zheng \& Bennett, 2002). Segundo esses autores, quanto maior a escala utilizada no experimento, maior a possibilidade de haver heterogeneidade na condutividade hidráulica e na porosidade do solo, que são consideradas homogêneas ao longo do percurso na equação de transporte convectivo-dispersivo. A 


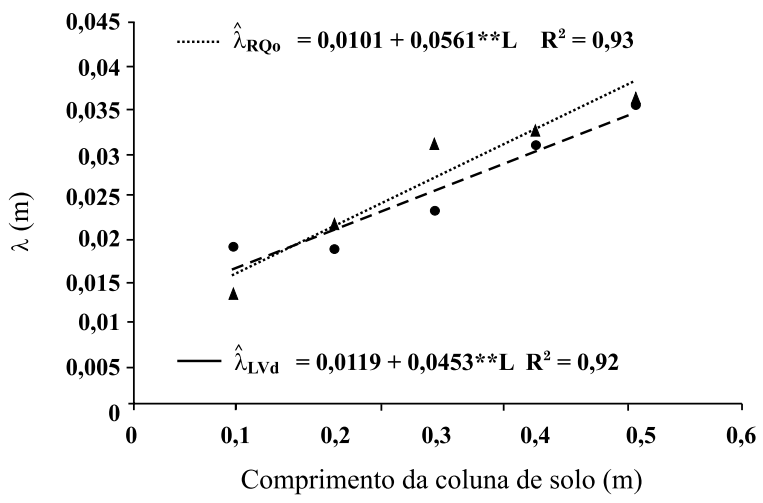

Figura 5. Dispersividades $(\lambda)$ do potássio estimadas (linhas) e médias dos valores experimentais observados num Neossolo Quartzarênico órtico (A) e num Latossolo Vermelho distrófico (O), em função do comprimento da coluna de solo (L). **: significativo a $1 \%$ pelo teste $t$.

diminuição da uniformidade no preenchimento das colunas à medida que se aumenta o comprimento pode provocar aumento na heterogeneidade macroscópica do meio poroso e, com isso, aumentar a dispersividade.

Em alguns trabalhos não foi observada a influência do comprimento da coluna sobre a $\lambda$. Shukla et al. (2003) verificaram que não houve relação dos valores de $\lambda$ com o comprimento da coluna testando colunas variando de 0,10 a $0,30 \mathrm{~m}$ de comprimento, preenchidas com solo desestruturado (franco e franco-arenoso) sob condição de saturação. Khan \& Jury (1990) também não observaram relação da $\lambda$ com o comprimento das colunas de solo desestruturado de 0,87, 0,43 e 0,22 m. Contudo, no trabalho de Shukla et al. (2003) a variação no comprimento foi pequena, e no de Khan \& Jury (1990) as repetições foram poucas.

De acordo com os resultados, observa-se que há necessidade de estabelecer uma padronização em relação ao comprimento da coluna e à velocidade de escoamento da solução aplicada ao solo. A padronização da velocidade de escoamento da solução é difícil de obter; o melhor seria trabalhar com a velocidade observada no solo em sua condição natural, obtida por teste de infiltração no campo. Quanto ao comprimento da coluna, o seu aumento desfavorece a uniformidade no preenchimento. Lewis \& Sjöstrom (2010) recomendam o padrão sugerido por Bergström (2000) para colunas cilíndricas de solo insaturado, que é uma relação de 1:4 do diâmetro com o comprimento da coluna.

\section{CONCLUSÕES}

Houve relação do comprimento da coluna e da velocidade de escoamento da solução com os parâmetros de transporte do $\mathrm{K}^{+}$tanto no Latossolo Vermelho distrófico quanto no Neossolo Quartzarênico órtico. No $\mathrm{LVd}$, o fator de retardamento aumentou com o aumento na velocidade de escoamento da solução. Em ambos os solos, o fator de retardamento diminuiu com o aumento no comprimento da coluna, o coeficiente de dispersão-difusão aumentou com o incremento na velocidade de escoamento da solução e com o aumento no comprimento da coluna de solo e a dispersividade também aumentou com o aumento no comprimento da coluna de solo.

\section{AGRADECIMENTOS}

Ao CNPq pela concessão de bolsas de pós-graduação e de produtividade em pesquisa e à FAPEMIG pelo auxílio financeiro ao projeto.

\section{LITERATURA CITADA}

AKHTAR, M.S.; STEENHUIS, T.S.; RICHARDS, B.K. \& MCBRIDE, M.B. Chloride and lithium transport in large arrays of undisturbed silt loam and sandy loam soil columns. Vadose. Zone J., 2:715-727, 2003.

ALCÂNTARA, M.A.K. \& CAMARGO, O.A. Movimentação de nitrato em horizonte superficial e subsuperficial de Latossolo e Nitossolo com cargas variáveis. Pesq. Agropec. Bras., 45:81-88, 2010.

ÁLVAREZ-BENEDÍ. J.; ISLA, T.; CARTÓN, A. \& BOLADO, S. Efecto de la velocidad de flujo en los parámetros de transporte de solutos. In: MUÑOZ-CARPENA, R.; RITTER, A. \& TASCÓN, C., eds. Estudios de la Zona No Saturada del Suelo. Laguna, ICIA, 1999. p.157-161.

ANAMI, M.H.; SAMPAIO, S.C.; SUSZEK, M.; GOMES, S.D. \& QUEIROZ, M.M.F. Deslocamento miscível de nitrato e fosfato proveniente de água residuária da suinocultura em colunas de solo. R. Bras. Eng. Amb., 12:75-80, 2008.

ARAÚJO, C.A.S.; RUIZ, H.A.; SILVA, D.J.; FERREIRA, P.A.; ALVAREZ V., V.H. \& BAHIA FILHO, A.F.C. Eluição de magnésio, cálcio e potássio de acordo com o tempo de difusão em colunas com agregados de um Latossolo Vermelho distrófico típico. R. Bras. Ci. Solo, 27:231-238, 2003.

BASHA, H.A. \& EL-HABEL, F.S. Analytical solution of the onedimensional time-dependent transport equation. Water Res. Res., 29:3209-3214, 1993.

BARIZON, R.R.M.; LAVORENTI, A.; REGITANO, J.B.; PRATA, F. \& TORNISIELO, V.L. Simulação do transporte e da sorção de imazaquim em colunas de solo. R. Bras. Ci. Solo, 30:615-623, 2006.

BEJAT, L.; PERFECT, E.; QUISENBERRY, V.L.; COYNE, M.S. \& HASZLER, G.R. Solute transport as related to soil structure in unsaturated intact soil blocks. Soil Sci. Soc. Am. J., 64:818-826, 2000. 
BERGSTRÖM, L. Leaching of agrochemicals in field lysimeters - a method to test mobility of chemicals in soil. In: CORNEJO, J.; JAMET, P. \& LOBNIK, F., eds. Pesticide/ Soil Interactions: Some Current Research Methods. INRA, Paris, p.479, 2000

BIGGAR, J.W. \& NIELSEN, D.R. Spatial variability of the leaching characteristics of a field soil. Water Res. Res., 12:78-84, 1976

BORGES JÚNIOR, J.C.F. \& FERREIRA, P.A. Equações e programa computacional para cálculo do transporte de solutos do solo. R. Bras. Eng. Agríc. Amb., 10:604-611, 2006.

BROMLY, M.; HINZ, C. \& AYLMORE, L.A.G. Relation of dispersivity to properties of homogeneous saturated repacked soil columns. Europ. J. Soil Sci., 58:293-301, 2007.

BRUSSEAU, M.L. The influence of solute size, pore-water velocity, and intraparticle porosity on solute dispersion and transport in soil. Water Res. Res., 29:1071-1080, 1993.

BUTTERS, G.L. \& JURY, W.A. Field scale transport of bromide in an unsaturated soil. 2. Dispersion modelling. Water Res. Res., 25:1583-1589, 1989.

CHU, Y.; JIN, Y.; BAUMANN, T. \& YATES, M.V. Effect of soil properties on saturated and unsaturated virus transport through columns. J. Environ. Quality, 32:2017-2025, 2003.

CORWIN, D.L. Evaluation of a simple lysimeter-design modification to minimize sidewall flow. J. Contam. Hydrol., 42:35-49, 2000

COSTA, J.L. \& PRUNTY, L. Solute transport in fine sandy loam soil under different flow rates. Agric. Water Manag., 83:111-118, 2006.

DEFELIPO, B.V. \& RIBEIRO, A.C. Análise química do solo (Metodologia). 2.ed. Viçosa, MG, Universidade Federal de Viçosa, 1977. 26p. (Boletim de Extensão, 29)

DONTSOVA, K.M.; YOST, S.L.; SIMUNEK, J.; PENNINGTON, J.C. \& WILLIFORD, C.W. Dissolution and transport of TNT, RDX, and Composition B in saturated soil columns. J. Environ. Quality, 35:2043-2054, 2006.

EMPRESA BRASILEIRA DE PESQUISA AGROPECUÁRIA . EMBRAPA. Centro Nascional de Pesquisa de Solos. Manual de análises de solo. Rio de Janeiro, 1997. 212p.

FERREIRA, P.A.; GARCIA, G.O.; MATOS, A.T.; RUIZ, H.R. \& BORGES JÚNIOR, J.C.F. Transporte no solo de solutos presentes na água residuária de café conilon. Acta Sci., Agron., 28:29-35, 2006.

GELHAR, L.W.; WELTY, C. \& REHFELDT, K.R. A critical review of data on field-scale dispersion in aquifers. Water Res. Res., 28:1955-1974, 1992.

GUBER, A.K.; SHELTON, D.R. \& PACHEPSKY, Y.A. Transport and retention of manure-borne coliforms in soil. Vadose Zone J., 4:828-837, 2005.

HUANG, K.; TORIDE, N. \& van GENUCHTEN, M.T. Experimental investigation of solute transport in large, homogeneous and heterogeneous, saturated soil columns. Transport Porous Media, 18:283-302, 1995.
JAYNES, D.B.; BOWMAN, R.S. \& RICE, R.C. Transport of a conservative tracer in the field under continuous flood irrigation. Soil Sci. Soc. Am. J., 52:618-624, 1988.

KHAN, A.U.H. \& JURY, W.A. A laboratory study of the dispersion scale effect in column outflow experiments. J. Contam. Hydrol., 5:119-131, 1990.

LAPIDUS, L. \& AMUNDSON, N.R. A descriptive theory of leaching. Mathematics of adsorption beds. J. Phys.Chem., 56:984-988, 1952

LEWIS, J. \& SJÖSTROM, J. Optimizing the experimental design of soil columns in saturated and unsaturated transport experiments. J. Contam. Hydrol., 115:1-13, 2010.

LI, Y. \& GHODRATI, M. Transport of nitrate in soils as affected by earthworm activities. J. Environ. Quality, 24:432-438, 1995.

LOBO, V.M.M.; RIBEIRO, A.C.F. \& VERISSIMO, L.M.P. Diffusion coefficients in aqueous solutions of potassium chloride at high and low concentrations. J. Molec. Liq., 78:139-149, 1998

MATOS, A.T.; COSTA, L.M.; FONTES, M.P.F. \& MARTINEZ, M.A. Retardation factors and the dispersion-diffusion coefficients of zinc, cadmium, copper and lead in soils from Viçosa MG, Brazil. Trans. ASAE, 42:903-910, 1999.

MELO, R.F.; FERREIRA, P.A.; MATOS, A.T.; RUIZ, H.A. \& OLIVEIRA, L.B. Deslocamento miscível de cátions básicos provenientes da água residuária de mandioca em colunas de solo. R. Bras. Eng. Agríc. Amb., 10:456-465, 2006.

MILFONT, M.L.; ANTONINO, A.C.D.; MARTINS, J.M.F.; NETTO, A.M.; GOUVEIA, E.R. \& CORREA, M.M. Transporte do paclobutrazol em colunas de solos. R. Bras. Ci. Solo, 32:2165-2175, 2008.

MONTOYA, J.C.; COSTA, J.L.; LIEDL, R.; BEDMAR, F. \& DANIEL, P. Effects of soil type and tillage practice on atrazine transport through intact soil cores. Geoderma, 137:161-173, 2006

NKEDI-KIZZA, P.; BIGGAR, J.W.; van GENUCHTEN, M.T.; WIERENGA, P.J.; SELIM, H.M.; DAVIDSON, J.M. \& NIELSEN, D.R. Modeling tritium and chloride 36 transport through an aggregated Oxisol. Water Res. Res., 19:691-700, 1983

OLIVEIRA, E.M.M.; RUIZ, H.A.; FERREIRA, P.A.; ALVAREZ V., V.H. \& BORGES JÚNIOR, J.C.F. Fatores de retardamento e coeficientes de dispersão-difusão de fosfato, potássio e amônio em solos de Minas Gerais. R. Bras. Eng. Agríc. Amb., 8:196-203, 2004.

RAYMUNDO, E.R.; IOURII, N.G.; DUWIG, C.; PANO, B.L.P.; MORENO, C.I.H.; REYES, F.G. \& SANDOVAL, B.F. Atrazine transport in a Mexican andosol and vertisol. Interciencia, 34:330-337, 2009.

ROCKHOLD, M.L.; YARWOOD, R.R.; NIEMET, M.R.; BOTTOMLEY, P.J. \& SELKER, J.S. Experimental observations and numerical modeling of coupled microbial and transport processes in variably saturated sand. Vadose Zone J., 4:407-417, 2005. 
RUIZ, H.A. Incremento da exatidão da análise granulométrica do solo por meio da coleta da suspensão (silte + argila). R. Bras. Ci. Solo, 29:297-300, 2005.

RUIZ, H.A.; FERREIRA, P.A.; ROCHA, G.C. \& BORGES JR, J.C.F. Transporte de solutos no solo. In: JONG van LIER, Q., ed. Física do solo. Viçosa, MG, Sociedade Brasileira de Ciência do Solo, 2010. p.213-240.

SCHULIN, R.; WIERENGA, P.J.; FLÜHLER H. \& LEUENBERGER, J. Solute transport through a Stony soil. Soil Sci. Soc. Am. J., 51:36-42, 1987.

SHUKLA, M.K.; ELLSWORTH, T.R.; HUDSON, R.J. \& NIELSEN, D.R. Effect of water flux on solute velocity and dispersion. Soil Sci. Soc. Am. J., 67:449-457, 2003.
SILVA, J.B.G. Tecnologia inovadora para retirada de amostras de solo com estrutura indeformada. Viçosa, MG, Universidade Federal de Viçosa, 2009. 57p. (Tese de Mestrado)

STOLF, R.; THURLER, A.M.; BACCHI, O.O.S. \& REICHARDT, K. Method to estimate soil macroporosity and microporosity based on sand content and bulk density. R. Bras. Ci. Solo, 35:447-459, 2011.

WIERENGA, P.J. \& van GENUCHTEN, M.T. Solute transport through small and large unsaturated soil columns. Ground Water, 27:35-42, 1989.

ZHENG, C. \& BENNETT, G.D. Applied contaminant transport modeling. 2.ed. New York, John Wiley \& Sons, 2002. $621 \mathrm{p}$. 